\title{
Parâmetros genéticos das características produtivas e reprodutivas de búfalos na Amazônia brasileira
}

\author{
Lisângela Aparecida Pinheiro Cassiano(1), Arthur da Silva Mariante ${ }^{(2)}$, Concepta McManus ${ }^{(1)}$, \\ José Ribamar Felipe Marques ${ }^{(3)}$ e Norton Amador da Costa ${ }^{(3)}$
}

\begin{abstract}
(1)Universidade de Brasília, Fac. de Agronomia e Medicina Veterinária, Caixa Postal 4508, CEP 70910-900 Brasília, DF. E-mail: cassiano@unb.br, concepta@unb.br (2)Embrapa Recursos Genéticos e Biotecnologia, Caixa Postal 02372, CEP 70770-900 Brasília, DF. E-mail: mariante@cenargen.embrapa.br (3)Embrapa Amazônia Oriental, Caixa Postal 48, CEP 66095-100 Belém, PA. E-mail: marques@cpatu.embrapa.br, norton@cpatu.embrapa.br
\end{abstract}

Resumo - O objetivo deste trabalho foi estimar componentes de variância e parâmetros genéticos, incluindo correlações genéticas, fenotípicas e herdabilidade das características produtivas e reprodutivas de búfalos na Amazônia brasileira. As estimativas de herdabilidade $\left(\mathrm{h}^{2}\right)$ das características variaram de 0,04 a 0,05 no primeiro intervalo entre partos, de 0,0 a 0,26 no intervalo entre partos, e de 0,0 a 0,25 no período de serviço, refletindo grande influência ambiental. Na idade ao primeiro parto, a herdabilidade variou de 0,12 a 0,38 . Em relação ao peso ao nascer, a raça Murrah foi a que apresentou a mais alta $\mathrm{h}^{2}(0,62)$. As correlações genéticas variaram de 1,00 a $-1,00$. Foram negativas as correlações genéticas entre idade ao primeiro parto e peso da fêmea adulta $(-0,12$ a -1,00 dependendo da raça), assim como as correlações entre período de serviço, peso ao nascer e peso da fêmea adulta $(0,01 \mathrm{a}-1,00)$. $\mathrm{O}$ efeito de ambiente permanente $\left(\mathrm{c}^{2}\right)$ variou de 0,000 a 0,155 . Somente no intervalo entre partos para a raça Jafarabadi pode-se considerar que houve $\mathrm{c}^{2}$ significativo $(0,458)$. O efeito materno $\left(\mathrm{m}^{2}\right)$ no peso ao nascer variou de baixo a médio, nas raças Carabao, Jafarabadi, Mediterrâneo e Murrah (0,11, 0,17, 0,37, 0,04, respectivamente).

Termos para indexação: correlações genéticas, herdabilidades, índices de produção e reprodução.

\section{Genetic parameters of production and reproduction traits of buffaloes in the Brazilian Amazon}

\begin{abstract}
The aim of this study was to estimate genetic parameters, including genetic and phenotypic correlations as well as heritabilities, for production and reproduction traits of buffaloes in the Brazilian Amazon. Heritability estimates varied from 0.04 to 0.05 for $\mathrm{FCI}$ (first calving interval), 0.0 to 0.26 for CI (calving interval) and 0.0 to 0.25 for SP (service period), reflecting high environmental variation for these traits. For AFC (age at first calving) the heritabilities varied from 0.12 to 0.38 . The Murrah breed showed the highest heritabilitiy for BW (birth weight) (0.62). Genetic correlations varied between 1.00 and -1.00 depending on the traits. There were negative genetic correlations between AFC and MFW (mature female weight) (-0.12 to -1.00 depending on breed) as well as between SP, BW and MFW (0.01 to -1.00). Permanent environment effects of the traits were low (0.000 to 0.155$)$ and only that for $\mathrm{CI}$ in the Jafarabadi breed could be considered significant (0.458). Maternal effects for birth weight were low to medium $(0.11,0.17,0.37$ and 0.04 for Carabao, Jaffarabadi, Mediterranean and Murrah, respectively).
\end{abstract}

Index terms: genetic correlations, heritabilities, production and reproduction indices.

\section{Introdução}

Dos bubalinos introduzidos no Brasil, quatro raças são reconhecidas oficialmente pela Associação Brasileira de Criadores de Búfalos: Carabao, Jafarabadi, Mediterrânea e Murrah, e, com exceção da Mediterrânea, todas as demais são de origem asiática. Os bubalinos adaptaram-se bem à Região Norte, em razão da semelhança das condições ambientais locais com as de seus países de origem, caracterizadas pelo clima predominantemente tropical. Desde então, os bubalinos vêm sendo essenciais para a pecuária de corte e de leite no país, especialmente na Região Norte, onde se encontra cerca de 50\% do rebanho bubalino brasileiro (IBGE, 2002).

A necessidade de se conhecer o potencial genético do rebanho bubalino decorre da sua importância econômica, não apenas na Região Norte, mas em todo o país. 
A coleta de informações sobre os componentes genéticos de cada raça é primordial, sobretudo da raça Carabao, submetida a um alto risco de extinção, principalmente em virtude dos cruzamentos indiscriminados entre raças. Segundo Silveira (2001), o conhecimento de parâmetros genéticos é necessário na estimativa de valores genéticos, na combinação de características em índices de seleção, na otimização de esquema de seleção, bem como na previsão de respostas à seleção.

A importância das características reprodutivas, em programas de melhoramento genético, está relacionada principalmente com as taxas de ganho genético anual. Menores idades ao primeiro parto permitem a redução do intervalo de gerações, enquanto menores períodos de serviço e menores intervalos entre partos disponibilizam maiores números de novilhas de alto potencial genético que possam substituir as fêmeas sendo descartadas. Segundo Gaines (1994), citado por Leite et al. (2001), o ganho potencial resultante do incremento na taxa reprodutiva é cinco vezes maior que o esperado pelo aumento da qualidade do leite e três vezes maior que o esperado pelo melhoramento genético, sendo apenas inferior aos ganhos que podem ser obtidos pela melhoria na nutrição. Silveira (2001), no entanto, afirma que para que se possa ter sucesso no melhoramento das características de importância econômica, fazse necessário conhecer os fatores ambientais ou as fontes da variação não-genéticas que atuam sobre as mesmas, e que interferem no crescimento e desenvolvimento dos rebanhos.

Apesar de sua relevância, poucos são os estudos sobre os efeitos genéticos e ambientais que afetam a eficiência reprodutiva dos bubalinos, principalmente no Brasil. Aziz et al. (2001), no Egito, verificaram que os búfalos apresentaram longos intervalos de partos e longos períodos de serviço. Segundo esses autores, as estimativas muito baixas de herdabilidade coincidiram com baixas correlações genéticas e moderadas correlações fenotípicas entre as características, ou seja, as características estudadas foram mais influenciadas pelos fatores não genéticos. Desta forma, o mais eficiente para melhorar as características reprodutivas do rebanho seria apenas manejo mais eficiente, ou uma melhor alimentação, já que o rebanho pouco responderia à seleção, por suas baixas estimativas de herdabilidade, e baixas correlações genéticas e fenotípicas.

Mahdy et al. (1999), também no Egito, verificaram que a melhora no manejo é a solução economicamente viável para se obter melhora também no intervalo entre partos nesta espécie, pois encontraram uma herdabilidade de 0,17 , ou seja, apenas $17 \%$ dos fatores que influenciam essa característica são de ordem genética.

Em relação à característica idade ao primeiro parto de diversas raças de bubalinos criados na Índia, Taneja (1999) relatou que há grande variação entre as raças bubalinas criadas nos trópicos, especialmente quando se consideram as condições de manejo. Nos búfalos das raças Murrah, Nili-Ravi, Egípcia, Pandharpuri, Surti e Bhadawar, a estimativa de herdabilidade em relação ao intervalo entre partos varia entre 0,26 e 0,37 . Estes valores revelam que, para aquelas raças, esta característica responderia a um programa de seleção.

O objetivo deste trabalho foi estimar componentes de variância e parâmetros genéticos, incluindo correlações genéticas, fenotípicas e herdabilidade das características produtivas e reprodutivas de búfalos na Amazônia brasileira.

\section{Material e Métodos}

O trabalho foi realizado em rebanhos de búfalos das raças Carabao (237 registros), Jafarabadi (245 registros), Mediterrâneo (1975 registros) e Murrah (427 registros), com características genéticas e fenotípicas particulares, perfazendo um total de 2.884 registros.

Os animais foram criados extensivamente, em sistema de conservação on farm, em pastagens nativas de terra inundável, dividido em vários retiros, em cuja composição botânica predominam gramíneas dos gêneros Paspalum, Luziola, Echinochose, Leersia, Hymenachne, Oriza, Panicum e Brachiaria. O sistema de acasalamento foi o da monta natural a campo. Foram adotadas práticas de sanidade que abrangiam vacinações e combate aos ecto e endo-parasitas.

Os rebanhos de bubalinos estudados pertencem à Embrapa Amazônia Oriental, e foram avaliados no período de 1977 a 1995 no Campo Experimental do Baixo Amazonas, situado à margem esquerda do Rio Amazonas, Município de Monte Alegre, PA, cujas coordenadas são $2^{\circ} 0^{\prime} 30^{\prime \prime}$ Sul e $54^{\circ} 4^{\prime} 13^{\prime \prime}$ Oeste, e uma altitude de $30 \mathrm{~m}$. O tipo climático é caracterizado como Ami, ou seja, tropical chuvoso, com pequeno período seco. A temperatura média anual é de $26,6^{\circ} \mathrm{C}$, variando entre $22,6^{\circ} \mathrm{C}$ e $31,2^{\circ} \mathrm{C}$. A umidade relativa do ar é, em média, $84 \%$, a precipitação pluviométrica anual é de $2.100 \mathrm{~mm}$, e a insolação anual total é de 2.091,5 horas. As principais 
unidades de relevo são as planícies e baixos planaltos amazônicos, e os solos da região pertencem aos grupos Glei Húmico Orgânico e Meio Orgânico.

$\mathrm{Na}$ estimativa da herdabilidade e das correlações genéticas, fenotípicas e ambientes, utilizou-se o método de máxima verossimilhança restrita livre de derivadas para o modelo animal, MTDFREML (Multiple Trait Derivate Free Restricted Maximum Likelihood), desenvolvido por Boldman et al. (1995):

$\mathrm{Y}=\mathrm{Xb}+\mathrm{Z}_{1} \mathrm{a}+\mathrm{Z}_{2} \mathrm{~m}+\mathrm{Z}_{3} \mathrm{pe}+\mathrm{e}$

em que: Y é o vetor das observações (IEP ${ }_{1}$, IPP, PS, IEP e PN); b é o vetor dos efeitos fixos (ano, mês, sexo do bezerro e ordem de parição); $Z_{1}$ é a matriz de incidência dos efeitos genéticos aditivo diretos; a é o vetor de efeitos genéticos aditivo diretos do animal; $Z_{2}$ é a matriz de incidência dos efeitos genéticos aditivos maternos; $m$ é o vetor de efeito genético aditivo materno; $Z_{3}$ é a matriz de incidência do efeito do ambiente permanente; pe é o efeito ambiente permanente; e é o vetor dos erros aleatórios associados às observações.

A análise incorporou um "Individual Animal Model" (IAM) com um "Numerator Relationship Matrix" (NRM) usando os procedimentos do tipo "Restricted Maximum Likelihood" (REML).

Foram avaliadas a herdabilidade, o efeito do ambiente permanente e materno, e seus respectivos componentes de variância, e as correlações genéticas e fenotípicas. Esses procedimentos usam a chamada técnica da "Best Linear Unbiased Prediction" (BLUP) para efeitos fixos e a predição de efeitos variáveis.

Nas fêmeas, foram analisadas as seguintes características: peso da fêmea adulta (PFA), primeiro intervalo entre partos $\left(\mathrm{IEP}_{1}\right)$, período de serviço (PS), idade ao primeiro parto (IPP), intervalo entre partos (IEP). Nos bezerros foi analisado somente o peso ao nascer $(\mathrm{PN})$.

\section{Resultados e Discussão}

A estimativa de herdabilidade $\left(\mathrm{h}^{2}\right)$ para idade ao primeiro parto encontrada na raça Murrah de 0,24 (Tabela 1) foi inferior ao valor de 0,62 encontrado por Penchev (1998), para a mesma raça, na Bulgária. Em ambos os casos, esta característica responderia a um programa de seleção.

Taneja (1999) relata que há grande variação na idade ao primeiro parto (IPP) entre as raças bubalinas criadas nos trópicos, especialmente quando se considera as condições de manejo. $\mathrm{O}$ autor afirma que a estimativa de $\mathrm{h}^{2}$ para IPP nas raças Surti e Bhadawar variou entre 0,26 e 0,37 , semelhante às herdabilidades de 0,24 e 0,38 estimadas para as raças Murrah e Jafarabadi no presente trabalho. As grandes diferenças de manejo diluíram as influências genéticas no rebanho, no entanto, para melhorar o índice da IPP, bastaria uma melhora nas condições de manejo.

De modo geral, se estabelece um peso mínimo para as novilhas serem cobertas ou inseminadas pela primeira vez, o que faz com que novilhas que atinjam esse peso mais cedo, apresentem o primeiro parto também mais cedo. Essa característica deve estar, portanto, relacionada às estimativas de $\mathrm{h}^{2}$ de IPP com as estimativas de $\mathrm{h}^{2}$ do PN ao PFA. Neste trabalho, as correlações genéticas entre IPP e PFA foram negativas para todas as raças e não houve correlações fenotípicas para estas características (Tabela 2). Com o PN, as correlações genéticas foram positivas para todas as raças, podendo-se afirmar que ao selecionar em relação ao aumento no PN, está se aumentando também o IEP, o que de fato foi observado por Mariante \& Zancaner (1985), em um rebanho da raça Nelore, criado no Estado de São Paulo.

As correlações genéticas das características de IPP com IEP apresentaram resultados iguais a $-0,11,-0,56 \mathrm{e}$ 0,00 , respectivamente, para as raças Carabao, Mediterrâneo e Murrah. Em relação à raça Jafarabadi, no entanto, a correlação genética entre essas duas características foi estimada em 1,00, entre IEP e IPP, mais próxima do resultado $(0,47)$ encontrado por Najera et al. (1991). Quando as correlações genéticas foram calculadas entre IPP e IEP 1 , as estimativas encontradas foram de $0,08,-0,89,-1,00$ e $-0,82$, respectivamente, para as raças Carabao, Jafarabadi, Mediterrâneo e Murrah, ou seja, todas negativas ou altas, com exceção da primeira. Resultado semelhante foi encontrado por Silveira (2001), com um valor de $-0,69$ em bovinos da raça Nelore. Todos esses resultados indicam que um trabalho de seleção no sentido de diminuir a IPP poderia estar afetando negativamente a produção, já que aumentaria os IEPs. Desta forma, deve-se ter cautela para saber até onde compensa reduzir o IPP, sem prejudicar o IEP.

A característica intervalo entre partos foi avaliada por ocasião do primeiro intervalo entre partos ( $\left.\operatorname{IEP}_{1}\right)$, e nos intervalos entre partos subseqüentes (IEP). As estimativas de $\mathrm{h}^{2}$ para característica de $\mathrm{IEP}_{1}$ foram muito semelhantes para as diferentes raças (Tabela 1), e extremamente influenciadas pelo ambiente. O IEP, que 
apresentou estimativas de $h^{2}$ iguais a 0,00 , para as raças Carabao, Jafarabadi e Mediterrâneo, refletindo também a grande influência ambiental nesta característica, maior até em relação ao $\mathrm{IEP}_{1}$. Portanto, manejo adequado é fundamental para o sucesso da criação de búfalos.

Contudo, na raça Murrah, a estimativa da $\mathrm{h}^{2}$ para IEP foi de 0,26 , um pouco maior do que a relatada $(0,157)$ por Penchev (1998), em uma população de búfalas da raça Murrah na Bulgária. Este resultado é também bem superior ao encontrado $(0,17)$ por Mahdy $(1999)$. O autor afirma que é economicamente viável apenas a melhora no manejo, com o que concorda Aziz et al. (2001), que encontrou $\mathrm{h}^{2}$ para IEP de 0,07 em búfalos egípcios.

O IEP foi a única característica que apresentou efeito permanente de ambiente $\left(\mathrm{c}^{2}\right)$ diferente de zero em todas as raças (Tabela 1).

As correlações genéticas entre IEP e PN foram negativas nas raças Jafarabadi e Carabao, possivelmente pelo fato de as búfalas que pariram bezerros mais pesados serem exatamente aquelas que estavam em melhor estado nutricional (Tabela 2). No entanto, nas raças

Tabela 1. Estimativas dos componentes das variâncias genéticas diretas $\left(\sigma_{\mathrm{a}}^{2}\right)$, maternas $\left(\sigma_{\mathrm{m}}^{2}\right)$, ambiente permanente $\left(\sigma_{\mathrm{c}}^{2}\right)$, ambientais $\left(\sigma^{2}\right.$ ), fenotípicas $\left(\sigma_{\mathrm{p}}^{2}\right)$ e parâmetros genéticos de peso ao nascer $(\mathrm{PN})$, primeiro intervalo entre partos (IEP $\left.{ }_{1}\right)$, período de serviço (PS), idade ao primeiro parto (IPP) e intervalo entre partos (IEP) das quatro raças bubalinas estudadas.

\begin{tabular}{|c|c|c|c|c|c|}
\hline Estimativa & PN & $\mathrm{IEP}_{1}$ & PS & IPP & IEP \\
\hline & & & Carabao & & \\
\hline$\sigma_{\mathrm{a}}^{2}$ & 8,04 & $1.885,41$ & 268,03 & 407,43 & 0,00 \\
\hline$\sigma_{\mathrm{m}}^{\mathrm{a}^{\mathrm{a}}}$ & 2,25 & - & - & - & - \\
\hline$\sigma_{c}^{2}$ & 0,00 & - & 3,24 & - & 14,75 \\
\hline$\sigma_{\mathrm{e}}^{2}$ & 6,05 & $45.249,84$ & $6.144,54$ & $2.845,80$ & 745,15 \\
\hline$\sigma_{p}^{e_{p}^{e}}$ & 20,56 & $47.135,25$ & $6.415,82$ & $3.277,42$ & 759,91 \\
\hline$h^{2^{p}}$ & 0,39 & 0,04 & 0,04 & 0,12 & 0,00 \\
\hline $\mathrm{m}^{2}$ & 0,11 & - & - & - & - \\
\hline$c^{2}$ & 0,00 & - & 0,00 & - & 0,02 \\
\hline \multirow[t]{2}{*}{$\mathrm{e}^{2}$} & 0,29 & 0,96 & 0,96 & 0,87 & 0,98 \\
\hline & & & Jafarabadi & & \\
\hline$\sigma_{\mathrm{a}}^{2}$ & 6,20 & $3.947,14$ & 0,06 & 5,00 & 0,01 \\
\hline$\sigma_{\mathrm{m}}^{\mathrm{a}^{\mathrm{a}}}$ & 3,76 & - & - & - & - \\
\hline$\sigma_{c}^{2}$ & 0,00 & - & 556,67 & - & 18,11 \\
\hline$\sigma_{e}^{c_{e}^{c}}$ & 7,60 & $74.995,66$ & $3.018,27$ & 7,00 & 377,06 \\
\hline$\sigma_{p}^{e}$ & 22,30 & $78.942,80$ & $3.575,00$ & 13,20 & 395,18 \\
\hline$h^{2^{p}}$ & 0,28 & 0,05 & 0,00 & 0,38 & 0,00 \\
\hline $\mathrm{m}^{2}$ & 0,17 & - & - & - & - \\
\hline$c^{2}$ & 0,00 & - & 0,155 & - & 0,458 \\
\hline \multirow[t]{2}{*}{$\mathrm{e}^{2}$} & 0,34 & 0,95 & 0,84 & 0,53 & 0,95 \\
\hline & & & Mediterrâneo & & \\
\hline$\sigma_{\mathrm{a}}^{2}$ & 3,18 & $1.395,49$ & 268,03 & 407,43 & 0,00 \\
\hline$\sigma_{\mathrm{m}}^{2}$ & 7,34 & - & - & - & - \\
\hline$\sigma_{c}^{2}$ & 0,00 & - & 3,24 & - & 14,75 \\
\hline$\sigma_{e}^{c}$ & 4,40 & $33.491,76$ & $6.144,59$ & $2.845,80$ & 745,15 \\
\hline$\sigma_{p}^{e}$ & 19,74 & $34.887,25$ & $6.415,82$ & $3.277,42$ & 759,91 \\
\hline$h^{2^{p}}$ & 0,16 & 0,04 & 0,04 & 0,12 & 0,00 \\
\hline $\mathrm{m}^{2}$ & 0,37 & - & - & - & - \\
\hline$c^{2}$ & 0,00 & - & 0,00 & - & 0,02 \\
\hline$\underline{\mathrm{e}^{2}}$ & 0,22 & 0,96 & 0,66 & 0,87 & 0,98 \\
\hline & & & Murrah & & \\
\hline$\sigma_{\mathrm{a}}^{2}$ & 11,26 & $2.799,48$ & $1.868,34$ & 250,15 & 179,38 \\
\hline$\sigma_{m}^{2}$ & 0,70 & - & - & - & - \\
\hline$\sigma_{c}^{2}$ & 0,23 & - & 0,00 & 2,16 & 0,001 \\
\hline$\sigma_{\mathrm{e}}^{2}$ & 3,28 & $67.187,52$ & $5.677,01$ & 781,71 & 502,59 \\
\hline$\sigma_{p}^{2}$ & 18,28 & $69.987,00$ & $7.545,86$ & $1.034,02$ & 681,97 \\
\hline$h^{2^{p}}$ & 0,62 & 0,04 & 0,25 & 0,24 & 0,26 \\
\hline $\mathrm{m}^{2}$ & 0,04 & - & - & - & - \\
\hline$c^{2}$ & 0,13 & - & 0,00 & - & 0,15 \\
\hline $\mathrm{e}^{2}$ & 0,18 & 0,96 & 0,75 & 0,76 & 0,74 \\
\hline
\end{tabular}


Murrah e Mediterrâneo, essa correlação entre IEP e PN foi positiva, o que está de acordo com os dados de Mariante \& Zancaner (1985), obtidos num rebanho Nelore. Todas as correlações genéticas que envolviam a característica IEP ${ }_{1}$ com IEP e IPP foram negativas ou muito baixas nas raças estudadas. Este resultado deveu-se, possivelmente, ao fato de búfalas que pariram mais cedo, terem concebido precocemente, ou seja, as búfalas primíparas ainda se encontrarem em fase de crescimento corporal, o que ocasionou um aumento no $\mathrm{IEP}_{1}$. Assim como IEPs curtos são seguidos de IEPs mais longos na próxima estação (Bourdon \& Brinks, 1983), a correlação genética do IEP $_{1}$ com IEP foi negativa em todas as raças, apesar de suas correlações fenotípicas terem todas apresentado valores iguais a 1,00.

$\mathrm{A} \mathrm{h}^{2}$ para o período de serviço (PS) foi, em geral, muito baixa nas quatro raças estudadas, principalmente na Jafarabadi que apresentou $\mathrm{h}^{2}$ igual a 0,0 (Tabela 1).
Embora a Murrah tenha apresentado estimativa mais alta, de 0,25 , este resultado pouco reflete a interferência genético aditiva para esta característica. $\mathrm{O} \mathrm{c}^{2}$ para esta característica foi nulo nas raças estudadas.

De acordo com Silva et al. (2001), o mesmo foi estimado para o IEP no ecótipo Mantiqueira. Baixas estimativas de herdabilidade para esta característica estão de acordo com a maioria dos valores apresentados em trabalhos publicados em países de clima tropical e temperado (Itália, Brasil, Egito e Índia). Esta característica é muito influenciada por fatores ambientais e genéticos não-aditivos. Entre as justificativas para esses baixos valores, os autores citam a ação da seleção natural.

As correlações genéticas entre o PS e o IEP foram positivas em todas as raças, o que já era esperado, pois com o aumento do PS, aumenta também o intervalo entre partos (Tabela 2). Esta característica apresentou correlações genéticas negativas ou muito baixas com PN,

Tabela 2. Correlações genéticas (abaixo da diagonal) e fenotípicas (acima da diagonal) entre características reprodutivas das raças Carabao, Jafarabadi, Mediterrâneo e Murrah ${ }^{(1)}$.

\begin{tabular}{|c|c|c|c|c|c|c|}
\hline Característica & PFA & $\mathrm{PN}$ & $\mathrm{IEP}_{1}$ & PS & IPP & IEP \\
\hline \multicolumn{7}{|c|}{ Carabao } \\
\hline PFA & & $-0,01$ & $-0,24$ & $-0,24$ & $-1,00$ & 0,00 \\
\hline PN & 1,00 & & 0,07 & 0,07 & $-0,43$ & 1,00 \\
\hline $\mathrm{IEP}_{1}$ & $-0,60$ & $-0,82$ & & 1,00 & $-0,05$ & 1,00 \\
\hline PS & $-0,60$ & $-1,00$ & $-1,00$ & & $-0,05$ & 1,00 \\
\hline IPP & $-0,19$ & 1,00 & 0,08 & 0,08 & & 0,18 \\
\hline IEP & $-0,04$ & $-0,56$ & $-0,94$ & 1,00 & $-0,11$ & \\
\hline \multicolumn{7}{|c|}{ Jafarabadi } \\
\hline PFA & & 0,54 & $-0,47$ & $-0,47$ & 0,00 & 0,00 \\
\hline $\mathrm{PN}$ & 1,00 & & $-0,10$ & $-0,10$ & 0,79 & 0,14 \\
\hline $\mathrm{IEP}_{1}$ & $-0,39$ & 1,00 & & 1,00 & $-0,41$ & 1,00 \\
\hline PS & $-0,39$ & $-1,00$ & 1,00 & & $-0,41$ & 1,00 \\
\hline IPP & $-0,57$ & 1,00 & $-0,89$ & $-0,54$ & & 0,33 \\
\hline IEP & 0,05 & $-0,38$ & $-1,00$ & 1,00 & 1,00 & \\
\hline \multicolumn{7}{|c|}{ Mediterrâneo } \\
\hline PFA & & 0,60 & $-0,34$ & $-0,34$ & 0,00 & 0,82 \\
\hline $\mathrm{PN}$ & 1,00 & & 0,00 & 0,00 & $-0,03$ & $-0,02$ \\
\hline $\mathrm{IEP}_{1}$ & $-0,15$ & 0,05 & & 1,00 & 0,17 & 1,00 \\
\hline PS & $-0,22$ & $-1,00$ & $-1,00$ & & 0,17 & 1,00 \\
\hline IPP & $-1,00$ & 0,96 & $-1,00$ & $-1,00$ & & $-0,27$ \\
\hline IEP & $-0,21$ & 1,00 & $-0,81$ & 1,00 & $-0,56$ & \\
\hline \multicolumn{7}{|c|}{ Murrah } \\
\hline PFA & & 0,41 & $-0,09$ & $-0,09$ & 0,00 & $-0,54$ \\
\hline $\mathrm{PN}$ & $-0,22$ & & 0,07 & 0,07 & $-0,30$ & 0,14 \\
\hline $\mathrm{IEP}_{1}$ & 0,01 & 0,09 & & 1,00 & $-0,29$ & 1,00 \\
\hline PS & 0,01 & 0,09 & $-0,28$ & & $-0,29$ & 1,00 \\
\hline IPP & $-0,12$ & 0,97 & $-0,82$ & $-0,82$ & & $-0,29$ \\
\hline IEP & $-0,13$ & 0,42 & $-0,25$ & 0,25 & 0,00 & \\
\hline
\end{tabular}

(1)PFA: peso da fêmea adulta; PN: peso ao nascer; IEP $_{1}$ : primeiro intervalo entre partos; PS: período de serviço; IPP: idade ao primeiro parto; IEP: intervalo entre partos. 
pois fêmeas bem nutridas costumam parir bezerros maiores, e não atrasam seu ciclo reprodutivo.

Em relação à característica peso ao nascer $(\mathrm{PN})$, a raça Murrah foi a que apresentou a maior estimativa de $\mathrm{h}^{2}$, superando em muito as demais raças, que apresentaram índices de moderados a baixo (Tabela 1). A raça Carabao apresentou $\mathrm{h}^{2}$ de 0,39 , ou seja, quase dois terços das diferenças estão relacionadas ao ambiente. Esta última raça, assim como a Murrah, responderiam bem a um trabalho de seleção para esta característica. As estimativas de $h^{2}$ do PN nas raças Jafarabadi e Mediterrânea foram, respectivamente, 0,28 e 0,16, próximas ao valor de 0,14 encontrado por Mahdy et al. (1999), em búfalos egípcios, indicando também que a maior parte da variação é conseqüência de fatores ambientais.

Estudando o efeito de fatores genéticos e de meio sobre os pesos de bovinos da raça Nelore, Souza \& Ramos (1995) relataram uma estimativa de $h^{2}$ para PN de 0,54 , valor muito próximo ao encontrado para a raça Murrah que, em geral, apresentou herdabilidades mais elevadas para todas as características, quando comparada às demais raças de bubalinos incluídas no presente trabalho. Este valor é relativamente superior à maioria dos valores encontrados para esta característica em trabalhos anteriores.

$\mathrm{O} \mathrm{c}^{2}$ foi nulo em todas as populações estudas para $\mathrm{PN}$, ou seja, a influência do ambiente permanente ainda no útero materno no peso dos bezerros, neste estudo, foi nula (Tabela 1). Apesar disso, houve baixo efeito materno $\left(\mathrm{m}^{2}\right)$ e essa característica variou de 0,11 na raça Carabao, 0,17 na Jafarabadi, 0,04 na Murrah, e um valor médio, em relação à raça Mediterrâneo de 0,37.

O PN apresentou correlações genéticas negativas com o IEP, somente nas raças Carabao e Jafarabadi (Tabela 2). As correlações fenotípicas foram muito baixas ou negativas, também para a maioria das características em todas as raças, exceto para o peso da fêmea adulta (PFA), o que já era esperado, uma vez que fêmeas maiores, em condições normais, costumam parir crias mais pesadas.

Em todas as raças estudadas, as correlações entre o PFA e a maioria das características foram negativas ou muito baixas, ou seja, uma fêmea bem nutrida tem seu potencial reprodutivo afetado positivamente (Tabela 2). Contudo, as correlações fenotípicas do PFA com as demais características foram positivas para todas as raças. Apenas quando se correlacionou essa caracte- rística com o PN, em relação à raça Carabao, que é a de menor porte, o resultado foi negativo.

$\mathrm{A} \mathrm{h}^{2}$ não foi estimada para esta característica por causa do reduzido número de observações que se dispunha para o PFA.

\section{Conclusões}

1. As características de reprodução, em geral, em búfalos na Região Amazônica, apresentam baixas estimativas de herdabilidade, portanto não devem responder à seleção, exceto para o peso ao nascer.

2. As correlações genéticas entre as características variam dependendo da raça estudada, mostrando a necessidade de avaliar cada população individualmente.

3. Efeitos maternais e de ambiente permanente são baixos ou nulos para a maior parte das características e raças estudadas, mostrando que a melhoria no ambiente influi na produtividade desses rebanhos.

\section{Referências}

AZIZ, M.A.; SCHOEMAN, S.J.; JORDAAN, G.F.; EL-CHAFIE, O.M.; MAHDY, A.T. Genetic and phenotypic variation of some reproductive traits in Egyptian buffalo. South African Journal of Animal Science, v.31, p.195-199, 2001.

BOLDMAN, K.G.; KRIESE, L.A.; VLECK, L.D. van; JASSEL, C.P. van; KACHMAN, S.D. A manual for use of MTDFREML: a set of programs to obtain estimates of variances and covariances [DRAFT]. Lincoln: United States Department of Agriculture , 1995. $120 \mathrm{p}$.

BOURDON, R.M.; BRINKS, J.S. Calving date versus calving interval as a reproductive measure in beef cattle. Journal of Animal Science, v.57, p.1412-1417, 1983.

IBGE (Rio de Janeiro, RJ). O Estado do Pará. Disponível em: <http://www.mre.gov.br/revista/numero01/pa-p.htm>. Acesso em: nov. 2002.

LEITE, T.E.; MORAES, J.C.F.; PIMENTEL, C.A. Eficiência reprodutiva em vacas leiteiras. Ciência Rural, v.31, p.467-472, 2001.

MAHDY, A.E.; EL-SHAFIE, O.M.; AYYAT, M.S. Genetic study and sire values for some economic traits in Egyptian buffaloes. Alexandria Journal of Agricultural Research, v.44, p.15-35, 1999.

MARIANTE, A. da S.; ZANCANER, A. Crescimento e reprodução do gado Nelore: visão do criador e do pesquisador. São Paulo: Ed. dos Criadores, 1985. 152p.

NAJERA, A.J.M.; OLIVEIRA, H.N.; PEREIRA, J.C.C. Efeitos genéticos e não genéticos sobre características ponderais de duas populações de raça Nelore. Arquivo Brasileiro de Medicina Veterinária e Zootecnia, v.1, p.81-92, 1991. 
PENCHEV, P. Phenotypic and genotypic evaluation of the buffalo population bred in Bulgaria. Bulgarian Journal of Agricultural Science, v.4, p.463-469, 1998.

SILVA, M.V.G.B. da; COBUCI, J.A.; FERREIRA, W.J.; GUARAGNA, G.P.; OLIVEIRA, P.R.P. de. Respostas correlacionadas em características reprodutivas do programa de melhoramento do ecótipo Mantiqueira para produção de leite. Revista Brasileira de Zootecnia, v.30, p.1228-1235, 2001.

SILVEIRA, J.C. Estudo da influência de fatores genéticos e ambientais sobre as características produtivas e reprodutivas em bovinos Nelore no Estado de Mato Grosso do Sul. 2001. 67p. Dissertação (Mestrado) - Universidade de Brasília, Brasília.

SOUZA, J.C.; RAMOS, A. de A. Efeitos de fatores genéticos e do meio sobre os pesos de bovinos da raça Nelore. Revista Brasileira de Zootecnia, v.24, p.164-172, 1995.

TANEJA, V.K. Dairy breeds and selection. In: FALVEY, L.; CHANTALAKHANA, C. (Ed.). Smallholder dairying in the Tropics. Nairobi: International Livestock Research Institute, 1999. $462 \mathrm{p}$.

Recebido em 4 de março de 2003 e aprovado em 2 de março de 2004 